\title{
Behavior of laminated shell composite with imperfect contact between the layers
}

\author{
David Guinovart-Sanjuán ${ }^{\mathrm{a}, *}$, Raffaella Rizzoni ${ }^{\mathrm{b}}$, Reinaldo Rodríguez-Ramos c , Raúl Guinovart-Díaz ${ }^{\mathrm{c}}$, \\ Julián Bravo-Castillero ${ }^{\mathrm{c}}$, Ransés Alfonso-Rodríguez ${ }^{\mathrm{a}}$, Frederic Lebon ${ }^{\mathrm{d}}$, Serge Dumont ${ }^{\mathrm{e}}$, Igor Sevostianov ${ }^{\mathrm{f}}$, \\ Federico J. Sabina ${ }^{g}$ \\ ${ }^{a}$ Department of Mathematics, University of Central Florida, 4393 Andromeda Loop N, Orlando, FL 32816, USA \\ ${ }^{\mathrm{b}}$ Dipartimento di Ingegneria, Università di Ferrara, Via Saragat 1, 44122 Ferrara, Italy \\ ' Departamento de Matemática, Universidad de La Habana, San Lazaro y L, 10400 La Habana, Cuba \\ d Aix-Marseille University, CNRS, Centrale Marseille, LMA, 4 Impasse Nikola Tesla, CS 40006, 13453 Marseille Cedex 13, France \\ e University of Nîmes, Place Gabriel Péri, 30000 Nîmes, France \\ ${ }^{\mathrm{f}}$ Department of Mechanical and Aerospace Engineering, New Mexico State University, P.O. Box 30001, Las Cruces, NM 88003, USA \\ ${ }^{g}$ Instituto de Investigaciones en Matemáticas Aplicadas y en Sistemas, Universidad Nacional Autónoma de México, Delegación Álvaro Obregón, Apartado Postal 20-726, \\ 01000 CDMX D.F., Mexico
}

\begin{abstract}
The paper focuses on the calculation of the effective elastic properties of a laminated composite shell with imperfect contact between the layers. To achieve this goal, first the two-scale asymptotic homogenization method (AHM) is applied to derive the solutions for the local problems and to obtain the effective elastic properties of a two-layer spherical shell with imperfect contact between the layers. The results are compared with the numerical solution obtained by finite elements method (FEM). The limit case of a laminate shell composite with perfect contact at the interface is recovered. Second, the elastic properties of a spherical heterogeneous structure with isotropic periodic microstructure and imperfect contact is analyzed with the spherical assemblage model (SAM). The homogenized equilibrium equation for a spherical composite is solved using AHM and the results are compared with the exact analytical solution obtained with SAM.
\end{abstract}

\section{Introduction}

Composite materials have emerged as the materials of choice in various branches of industry - aerospace, automotive, sport, etc. for increasing the performance and reducing the weight and cost. However, defects induced during the manufacturing process or accumulated due to environmental and operational loads lead to the reduction in the mechanical performance and material strength and are recognized as a general problem in this type of composites, [1]. Most typically, such defects can be found at the interfaces between the layers creating an imperfect contact condition, $[2,3]$.

The effect of the contact imperfectness on elastic properties of composites attracted the attention of researchers from 1970's, $[4,5]$. In [6-9], the authors obtained analytical expressions for the effective elastic properties of rectangular fibrous composites with

\footnotetext{
* Corresponding author.

E-mail address: davidgs1601@gmail.com (D. Guinovart-Sanjuán).
}

imperfect contact between the matrix and the reinforcement. On the other hand, the multilayered curvilinear shell structures have received special attention in the last years. In [10-13] several mathematical methods have been used to derive analytical expression for the elastic properties of laminated shell composites. As a particular case, in [14], the expression of the effective coefficients for a curvilinear shell composite with perfect contact at the interface is obtained.

Several mathematical models and techniques have been developed to evaluate the elastic properties of curvilinear laminated shell composites with imperfect contact at the interfaces. In papers as $[10,12,15-20]$, the assemblage model, finite elements method and the two-scale asymptotic homogenization method are used to derive in one way or another the effective behavior of the elastic properties of particular composites with imperfect contact at the interface.

In this paper a spherical shell structure is studied. In [21] the authors considered the effect in the elastic properties of a spherical laminated shell composite under the influence of stress and strain 
distributions for two composites with perfect and imperfect contact at the interface using AHM and SAM. In [21] the imperfect contact condition is modeled considering a thin interphase between the layers of the composite, i.e., a three phase composite is used in the analysis. Here the same effect in the spherical shell structure is studied except that the imperfect contact condition is modeled as a linear spring type and the FEM is used to validate the results obtained via AHM and SAM. The purpose of studying this kind of spherical structures obeys to the development and application of mathematical methods for the study of the cornea and other similar soft tissues.

In the present paper, first the AHM technique is used to evaluate the elastic properties of a two-layer laminated shell with imperfect contact of the spring type at the interface. The general analytical expressions of the effective coefficients are derived from the solution of the local problem. We focus on a two-layer spherical shell subjected to internal pressure assuming that the layers are isotropic. To validate the model, the effective coefficients of the spherical structure are compared with FEM calculations. The elastic fields (stresses, strains and displacements) are also compared with ones calculated by the method of Bufler [22] for the analysis of a spherical assemblage model (SAM). The approach is based on the transfer matrix method and yields closed form calculation of the equivalent elastic properties of a periodically laminated hollow sphere made of alternating layers of isotropic elastic materials with imperfect contact. The effective displacement, radial and hoop stresses computed via AHM are compared with the elastic fields calculated by FEM and SAM.

\section{The linear elastic problem}

A curvilinear elastic periodic composite is studied. The geometry of the structure is described by the curvilinear coordinates system $\mathbf{x}=\left(x_{1}, x_{2}, x_{3}\right) \in \Omega \subset \mathbb{R}^{3}$, where $\Omega=\Omega_{1} \cup \Omega_{2}$ is the region occupied by the solid, it is bounded by the surface $\partial \Omega=\Sigma_{1} \cup \Sigma_{2}$, where $\Sigma_{1} \cap \Sigma_{2}=\varnothing, \Omega_{\alpha} \alpha=1,2$ are the elements of the composite, separated by the interface $\Gamma^{\varepsilon}$. In $\Omega$, the stress $\sigma$ and strain $\epsilon$ are related through the Hooke's law, $\sigma^{i j}=C^{i j k l} \epsilon_{k l}$, where $C^{i j k l}$ are the components of the elastic tensor $\boldsymbol{C}$. For a linear periodic solid structure, the elastic tensor $\boldsymbol{C} \equiv \boldsymbol{C}(\mathbf{x}, \mathbf{y})$ is regular with respect to the slow variable $\mathbf{x}$ and $\mathbf{Y}$-periodic with respect to the fast variable $\mathbf{y}=\mathbf{x} / \varepsilon \in \mathbf{Y}$, where $0<\varepsilon \ll 1$ characterizes the periodicity of the composite and $\mathbf{Y}$ denotes the periodic cell.

The linear elastic equilibrium equation for a curvilinear laminated shell composite with imperfect contact (spring type) at the interface is

$\sigma_{j}^{i j}+\Gamma_{j k}^{i} \sigma^{k j}+\Gamma_{j k}^{j} \sigma^{i k}+f^{i}=0, \quad$ in $\Omega$,

subject to boundary conditions,

$u_{i}=u_{i}^{0} \quad$ on $\quad \Sigma_{1}, \quad \sigma^{i j} n_{j}=S^{i} \quad$ on $\quad \Sigma_{2}$,

and interface contact conditions,

$\sigma^{i j} n_{j}=K^{i j}\left[\left[u_{j}\right]\right], \quad\left[\left[\sigma^{i j} n_{j}\right]\right]=0, \quad$ on $\quad \Gamma^{\varepsilon}$,

where $\{\cdot\}_{j}=\frac{\partial}{\partial x_{j}}\{\cdot\}$ is the derivative with respect to the slow curvilinear coordinate, $\Gamma_{j k}^{i}$ are the Christoffel's symbols of second type, $[[\cdot]]=(\cdot)^{(2)}-(\cdot)^{(1)}$ denotes the jump at the interface $\Gamma^{\varepsilon}, n_{j}$ is the normal vector to the corresponding surface $\left(\Sigma_{2}, \Gamma^{\varepsilon}\right), K^{i j}$ are the components of a matrix $\mathbf{K}$, that characterizes the imperfect contact in $\Gamma^{\varepsilon}$ and the order of $\mathbf{K}$ is $O\left(\varepsilon^{-1}\right)$. Replacing the Hooke's law and considering the Cauchy's formula, $\epsilon_{i j}=\left(u_{i, j}+u_{j, i}\right) / 2$, the equations (1)-(3) can be rewritten for the displacement vector function [14].

\section{Homogenization of two-layer laminated shell composites with imperfect contact}

In order to obtain an equivalent problem to (1)-(3) with not fast oscillating coefficients, the two-scales Asymptotic Homogenization Method (AHM) is used. The general expression of the truncated expansion is given by

$u_{m}^{(\varepsilon)}=v_{m}+\varepsilon\left[\hat{N}_{m}^{p} v_{p}+N_{m}^{l k} v_{l, k}\right]+o(\varepsilon)$

where $v_{m} \equiv v_{m}(\mathbf{x}), N_{m}^{l k} \equiv N_{m}^{l k}(\mathbf{x}, \mathbf{y})$ is the local function for the first order approach, $N_{(1) m}^{l k}(\mathbf{x}, \mathbf{y})$ is $\mathbf{Y}$-periodic, where $\mathbf{Y}=[0,1]$ and $\hat{N}_{m}^{p}=-\Gamma_{l k}^{p} N_{m}^{l k}$ [14]. Substituting the expansion (4) into the Eqs. (1)-(3) a recurrent family of problem is obtained for different powers of the small parameter $\varepsilon$.

Considering a two-layer laminated shell composite with isotropic components, i.e.

$C^{i j k l}=\lambda(y) g^{i j} g^{k l}+\mu(y)\left(g^{l j} g^{k i}+g^{i l} g^{k j}\right)$,

where $\left[g^{i j}\right]$ is the metric tensor of the coordinates $\left(x_{1}, x_{2}, x_{3}\right)$ and

$\lambda(y)=\left\{\begin{array}{ll}\lambda_{1} & y \in[0, \gamma) \\ \lambda_{2} & y \in(\gamma, 1]\end{array}, \quad \mu(y)=\left\{\begin{array}{ll}\mu_{1} & y \in[0, \gamma) \\ \mu_{2} & y \in(\gamma, 1]\end{array}\right.\right.$,

where the layers are transversal to the axis $x_{3}$, the local problem is obtained for $\varepsilon^{-1}$

$\partial / \partial y\left(C^{i 3 k}+C^{i 3 m 3} \partial N_{m}^{k} / \partial y\right)=0 \quad$ on $\quad \mathbf{Y}=[0, \gamma) \cup\{\gamma\} \cup(\gamma, 1]$,

with interface conditions given by the expressions

$\left[C^{i 3 l k}+C^{i 3 m 3} \partial N_{(1) m}^{l k} / \partial y\right]=(-1)^{\alpha+1} K^{i j}\left[\left[N_{(1) j}^{l k}\right]\right] \quad$ on $\quad \Gamma^{\varepsilon}=\{y=\gamma\}$,

$\left[\left[C^{i 3 l k}+C^{i 3 m 3} \partial N_{(1) m}^{l k} / \partial y\right]\right]=0$

on $\Gamma^{\varepsilon}=\{y=\gamma\}$,

where the parameter $\alpha=1,2$ denotes the layer.

Substituting (5) into the local problem (6) the following expression is obtained $\partial^{2} N_{(1) m}^{l k} / \partial y^{2}=0$. Therefore, the local function has the expression

$N_{m}^{l k}= \begin{cases}A_{m}^{l k(1)} y+B_{m}^{l k(1)}, & y \in[0, \gamma), \\ A_{m}^{l k(2)} y+B_{m}^{l k(2)}, & y \in(\gamma, 1] .\end{cases}$

Considering the periodicity of the functions $N_{m}^{l k}$ and $\partial N_{m}^{l k} / \partial y$ the following linear equations system is obtained from Eq. (8)

$\left[C^{i 3 l k(1)}+C^{i 3 m 3(1)} A_{m}^{l k(1)}\right]=-K_{i m}\left(A_{m}^{l k(1)}(\gamma)+A_{m}^{l k(2)}(1-\gamma)\right)$,
$\left[C^{i 3 l k(2)}+C^{i 3 m 3(2)} A_{m}^{l k(2)}\right]=-K_{i m}\left(A_{m}^{l k(1)}(\gamma)+A_{m}^{l k(2)}(1-\gamma)\right)$,

where the supraindex $(\alpha) \alpha=1,2$ refers to each layer $\alpha$. The linear problem (10) and (11) related to the variables $A_{m}^{l k(\alpha)}$ can be solved using classical methods and therefore the local functions are obtained.

Applying the average operator to the coefficient of the parameter $\varepsilon^{0}$, the homogenized coefficients are obtained and the general expression is given in the Eqs. (12)-(18) of [14]. The effective coefficients for a two-layer laminated shell composite with isotropic layers and imperfect contact condition at the interface have the general analytic expression

$\widehat{h}^{i j k l}=\left\langle C^{i j k l}\right\rangle+V_{1} C^{i j m 3(1)} \frac{\partial N_{m}^{k l(1)}}{\partial y}+V_{2} C^{i j m 3(2)} \frac{\partial N_{m}^{k l(2)}}{\partial y}$.

where $V_{\alpha}$ is the volume of the layers of the composite and the local functions $\partial N_{m}^{k l(\alpha)} / \partial y$ have the expression 
$\frac{\partial N_{m}^{k l(\alpha)}}{\partial y}=\frac{-C^{q 3 k l(\alpha)}\left(K^{q n} V_{\beta}+C^{q 3 n 3(\beta)}\right)+C^{q 3 k l(\beta)} K^{q n} V_{\beta}}{C^{r 3 m 3(1)} C^{r 3 n 3(2)}+C^{r 3 m 3(1)} K^{r n} V_{2}+C^{r 3 n 3(2)} K^{r m} V_{1}}$,

for $\beta=1,2$ and $\beta \neq \alpha$.

The homogenized problem is obtained from the Eqs. (19) and (20) of [14].

\subsection{Comparison of the effective coefficients for composites with perfect and imperfect contact}

In order to illustrate the influence of the imperfect contact on the effective coefficients, a two layer rectangular laminate shell is considered (see Fig. (1)). The layers of the composite are isotropic and the materials are stainless steel (Young's modulus $E_{1}=206.74$ GPa, Poisson ratio, $v_{1}=0.3$ ) with volume $V_{1}$, represented by $Y_{1}$ on Fig. (1) and aluminum (Young's modulus $E_{2}=72.04 \mathrm{GPa}$, Poisson ratio, $v_{2}=0.35$ ) with volume $V_{2}=1-V_{1}$, represented by $Y_{2}$ on Fig. (1). The matrix $\mathbf{K}$ characterizes the imperfect contact at the interface $\Gamma$ (see Fig. (1)) and it has nonzero components $K^{11}=$ $K^{22}=\mu / \varepsilon \quad$ and $\quad K^{33}=(\mu+2 \lambda) / \varepsilon \quad$ where $\quad \mu=\lambda=1 \quad$ and $\varepsilon=[0.001,0.01,0.05]$. The effective coefficients for the imperfect contact case are calculated using the Eq. (12) and they are compared with the coefficients obtained using Eq. (26) of [14] for perfect contact case (see Fig. (2)). The convergence of effective

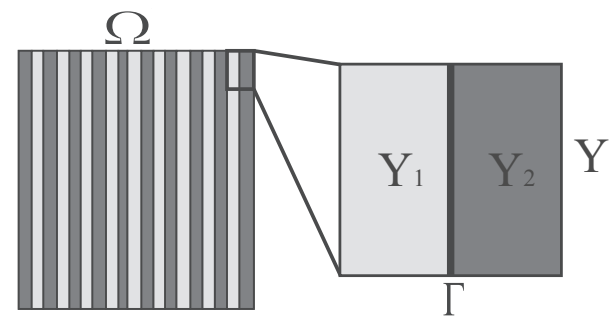

Fig. 1. Rectangular structure $\Omega$, unit cell $\mathbf{Y}$, the two elements $\mathbf{Y}_{1}$ and $\mathbf{Y}_{2}$ with the corresponding interface $\Gamma$. coefficients for the imperfect contact case can be appreciated as $\varepsilon \rightarrow 0$, i.e. $K^{i j} \rightarrow+\infty$.

For the case of $V_{1}=0$ or $V_{1}=1$, we are in presence of a monoelement multi-layered structure, i.e. it is a structure with several layers but each layer is the same element. It can be studied the perfect and the imperfect contact between the layers of these structures. For the perfect contact case, the mono-element multilayered structure are considered as an homogeneous structure. But a different situation is obtained for the imperfect contact case. Due to the imperfection, the structures are studied as a heterogeneous composite.

It can be seen in Fig. (2), for the perfect contact case, that the aluminum and stainless steel elastic moduli are reached for $V_{1}=0$ or $V_{1}=1$, respectively, as a whole homogeneous material. In particular, for $V_{1}=1$ the value $C^{3333}=h^{3333}=278.3038 \mathrm{GPa}$ is attained for stainless steel. In the case of imperfect contact the imperfection is modeled as a linear spring distributed across the common interface $\Gamma$ between the layers. When $K^{11}$ takes the values 1000,100 and 20, the elastic constant $h^{3333}$ is equal to 254.6779 , 144.3725 and $49.3587 \mathrm{GPa}$, respectively. Thus, the main effect of the imperfect contact is to degrade the composite's integrity: the value of the elastic constants diminishes accordingly, so a weakened composite is obtained. In other words, the particular case of $V_{1}=0\left(V_{1}=1\right)$ reports, for the imperfect contact case, the effective coefficients of the a structure $\Omega$ where the unit cell has two layers $Y_{1}$ and $Y_{2}$ made by aluminum (stainless steel), but with degrading due to the imperfection. It is very important to remark the fact that the AHM reports different values of the effective coefficients for mono-element multi-layers structures with perfect and imperfect contact at the interface; i.e. due to the imperfection, the effective coefficients of the structures with imperfect contact are weaker.

\section{The spherical assemblage model with imperfect contact (SAM)}

In this section, a spherical assemblage model consisting of $N$ different thin elastic layers is studied using the transfer-matrix method.
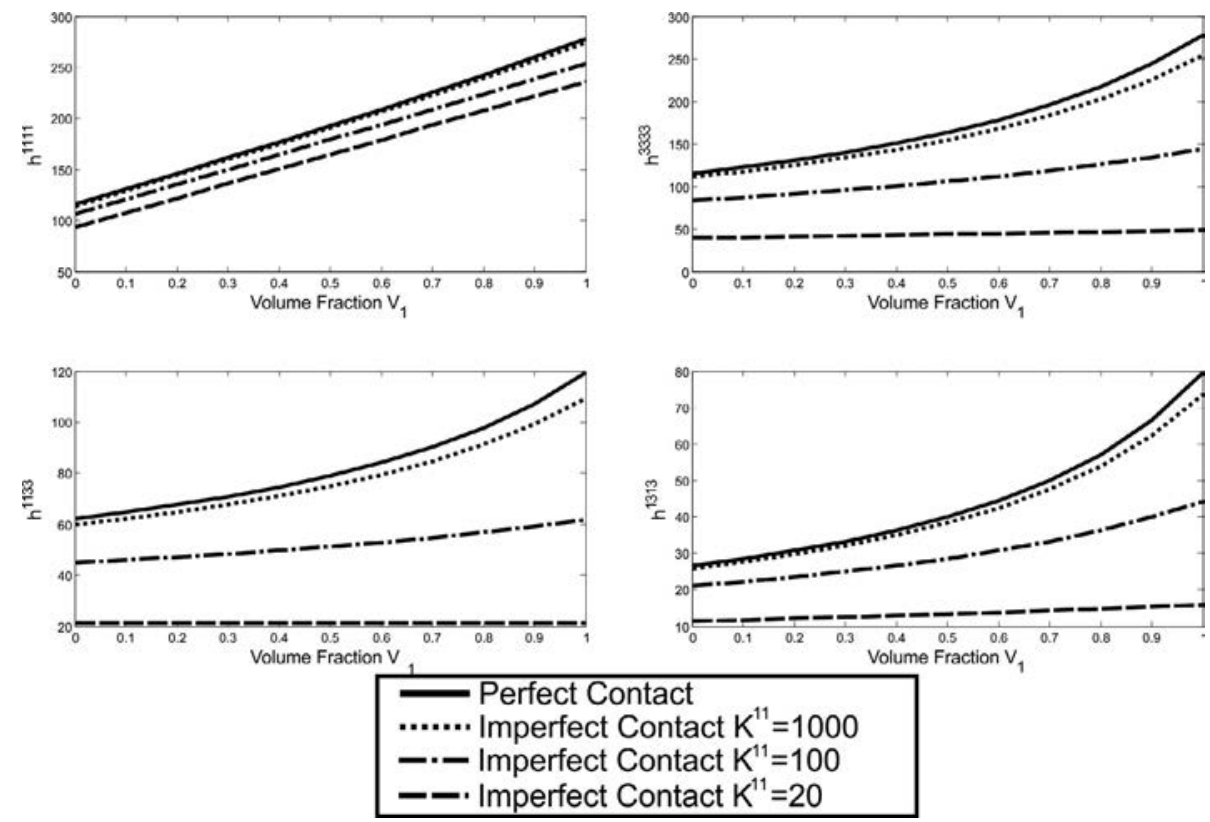

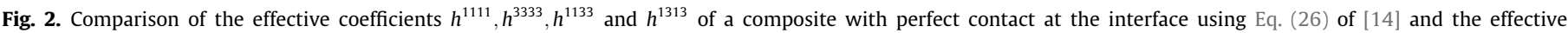
coefficients for a composite with imperfect contact using Eq. (12). 
The transfer-matrix method is a classic approach [23]. Here, we first review its application to a periodic laminated hollow sphere proposed in [22] and next extend the obtained results to the case of imperfect contact between the layers.

The spherical assemblage has internal radius $R_{i}$, external radius $R_{e}$ and thickness $h=2 t$. The inner surface $r=R_{i}$ is loaded by a constant pressure

$\sigma_{r r}\left(R_{i}\right)=+p$,

whereas the external surface $r=R_{e}$ is traction free

$\sigma_{r r}\left(R_{e}\right)=0$.

The $k$-th layer comprised between the radii $R_{k-1}$ and $R_{k}$, is characterized by the thickness $h_{k}$ and it is made of linear elastic homogeneous and isotropic material with Young modulus and Poisson ratio $E_{k}$ and $v_{k}$, respectively. According to the transfer-matrix method [22], the radial stress $\sigma_{r r}$ and displacement $u_{r}$ at radius $R_{k-1}$ of the laminated sphere can be related to the radial stress and displacement at radius $R_{k}$ through the field-transfer matrix $\mathbf{T}_{k}$ of the layer $k$ :

$\left[\begin{array}{c}\sigma_{r r}\left(R_{k}\right) \\ E^{*} u_{r}\left(R_{k}\right) / h^{*}\end{array}\right]=\mathbf{T}_{k}\left[\begin{array}{c}\sigma_{r r}\left(R_{k-1}\right) \\ E^{*} u_{r}\left(R_{k-1}\right) / h^{*}\end{array}\right]$,

$\mathbf{T}_{k}:=\left[\begin{array}{cc}1-a_{k} h & b_{k} h \\ c_{k} h & 1-d_{k} h\end{array}\right]$,

with

$a_{k}:=2\left(1-\frac{v_{k}}{\left(1-v_{k}\right)}\right) \frac{\lambda_{k}}{R_{i}}$

$b_{k}:=\frac{2}{\left(1-v_{k}\right)} \frac{E_{k} h^{*}}{E^{*}} \frac{\lambda_{k}}{R_{i}^{2}}$,

$c_{k}:=\left(1-\frac{2 v_{k}^{2}}{\left(1-v_{k}\right)}\right) \frac{E_{k} h^{*}}{E^{*}} \lambda_{k}$,

$d_{k}:=\frac{2 v_{k}}{\left(1-v_{k}\right)} \frac{\lambda_{k}}{R_{i}}$

Here, $\lambda_{k}=h_{k} / h$ is the thickness ratio of the $k$-th layer, and $E^{*}$ and $h^{*}$ denote a reference modulus of elasticity and thickness, respectively. Applying (16) N-times for the layered hollow sphere made of layers in perfect contact, we have

$\left[\begin{array}{c}\sigma_{r r}\left(R_{e}\right) \\ E^{*} u_{r}\left(R_{e}\right) / h^{*}\end{array}\right]=\mathbf{S}\left[\begin{array}{c}\sigma_{r r}\left(R_{i}\right) \\ E^{*} u_{r}\left(R_{i}\right) / h^{*}\end{array}\right], \quad \mathbf{S}:=\mathbf{T}_{N} \mathbf{T}_{N-1} \ldots \mathbf{T}_{1}$.

Here $\mathbf{S}$ is the transfer matrix system from radius $R_{i}$ to $R_{e}$, linking the two elastic states at the boundaries of laminated sphere.

Sustituting (16) into (22) and considering only the terms of order zero and one in $h$, Bufler's result is obtained:

$\mathbf{S}=\mathbf{I}-h \mathbf{M}+o(h), \quad \mathbf{M}:=\left[\begin{array}{ll}a & b \\ c & d\end{array}\right]$,

with

$a:=\sum_{k=1}^{N} 2\left(1-\frac{v_{k}}{\left(1-v_{k}\right)}\right) \frac{\lambda_{k}}{R_{i}}$

$b:=\sum_{k=1}^{N} \frac{2}{\left(1-v_{k}\right)} \frac{E_{k} h^{*}}{E^{*}} \frac{\lambda_{k}}{R_{i}^{2}}$,

$c:=\sum_{k=1}^{N}\left(1-\frac{2 v_{k}^{2}}{\left(1-v_{k}\right)}\right) \frac{E_{k} E^{*}}{h^{*}} \lambda_{k}$

$d:=\sum_{k=1}^{N} \frac{2 v_{k}}{\left(1-v_{k}\right)} \frac{\lambda_{k}}{R_{i}}$.

To extend these results to a laminated sphere with imperfect contact between the layers, an arrangement of springs is artificially considered at the spherical surface between adjacent layers. In this case, a jump of the radial displacement has to be taken into consideration, [24-27]. In particular, the continuity of radial stress is assumed and the following linear relation between the radial stress and the jump of radial displacement is imposed at the radius $R_{k}$ :

$\left[\begin{array}{c}\sigma_{r r}\left(R_{k}^{+}\right) \\ E^{*} u_{r}\left(R_{k}^{+}\right) / h^{*}\end{array}\right]=\hat{\mathbf{K}}_{k}\left[\begin{array}{c}\sigma_{r r}\left(R_{k}^{-}\right) \\ E^{*} u_{r}\left(R_{k}^{-}\right) / h^{*}\end{array}\right], \quad \hat{\mathbf{K}}_{k}:=\left[\begin{array}{cc}1 & 0 \\ E^{*} \varepsilon_{k} /\left(h^{*}\left(2 \mu_{k}+\lambda_{k}\right)\right) & 1\end{array}\right]$,

where the matrix $\hat{\mathbf{K}}_{k}$ characterizes the imperfect contact provided by the $k$-th layer of springs, with $k=1,2, \ldots N-1, \varepsilon_{k} \ll 1$ is a small length parameter accounting for its thickness and $2 \mu_{k}+\lambda_{k}$ its elasticity coefficient.

Now, the presence of springs are considered, therefore the transfer matrix system $\mathbf{S}$ (cf. (23)) modifies in order to incorporate the matrices $\hat{\mathbf{K}}_{k}$,

$\tilde{\mathbf{S}}=\mathbf{T}_{N} \hat{\mathbf{K}}_{N-1} \mathbf{T}_{N-1} \ldots \hat{\mathbf{K}}_{1} \mathbf{T}_{1}$.

Substituting (16) and (28) into (29), one obtains the new matrix system for a spherical assemblage made of $N$ layers with imperfect contact. It can be shown that

$\tilde{\mathbf{S}}=\mathbf{I}-h \mathbf{M}+o(h), \quad \tilde{\mathbf{M}}:=\left[\begin{array}{ll}a & b \\ \tilde{c} & d\end{array}\right]$,

with $a, b$ and $d$ given again by Eqs. (24), (25) and (27), respectively, and

$$
\begin{aligned}
\tilde{c} & :=c+\sum_{k=1}^{N-1} \frac{E^{*}}{h^{*}} \frac{\varepsilon_{k}}{\left(2 \mu_{k}+\lambda_{k}\right)} \\
& =\sum_{k=1}^{N}\left(1-\frac{2 v_{k}^{2}}{\left(1-v_{k}\right)}\right) \frac{E_{k} E^{*}}{h^{*}} \lambda_{k}+\sum_{k=1}^{N-1} \frac{E^{*}}{h^{*}} \frac{\varepsilon_{k}}{\left(2 \mu_{k}+\lambda_{k}\right)} .
\end{aligned}
$$

The case of a periodic laminate made by repeating $n$ times a group of $N$ layers is now considered. As $n$ increases, the thicknesses $h_{k}$ and $\varepsilon_{k}$ must decrease with $n$ in order to keep the total thickness $h$ of the fixed laminate. In particular, it is assumed that $h_{k}=\Lambda_{k} h / n$ and $\varepsilon_{k}=\xi_{k} h / n$, with $\Lambda_{k}, k=1,2, \ldots N$, the thickness ratio of the $k$ th layer inside the group, and analogously $\xi_{k} \ll 1$, $k=1,2, \ldots N-1$, for the $k$-th layer of springs. The matrix system for the hollow sphere with homogenized properties following Bufler is calculated as

$\lim _{h \rightarrow 0} \frac{1}{h}\left(\tilde{\mathbf{S}}^{n}-\mathbf{I}\right)=\lim _{h \rightarrow 0} \frac{1}{h}\left(\left(\mathbf{T}_{N} \hat{\mathbf{K}}_{N-1} \mathbf{T}_{N-1} \ldots \hat{\mathbf{K}}_{1} \mathbf{T}_{1}\right)^{n}-\mathbf{I}\right)=\langle\mathbf{M}\rangle$,

with

$\langle\mathbf{M}\rangle:=\left[\begin{array}{ll}\langle a\rangle & \langle b\rangle \\ \langle c\rangle & \langle d\rangle\end{array}\right]$

$\langle a\rangle:=\sum_{k=1}^{N} 2\left(1-\frac{v_{k}}{\left(1-v_{k}\right)}\right) \frac{\Lambda_{k}}{R_{i}}$,

$\langle b\rangle:=\sum_{k=1}^{N} \frac{2}{\left(1-v_{k}\right)} \frac{E_{k} h^{*}}{E^{*}} \frac{\Lambda_{k}}{R_{i}^{2}}$,

$\langle c\rangle:=\sum_{k=1}^{N}\left(1-\frac{2 v_{k}^{2}}{\left(1-v_{k}\right)}\right) \frac{E_{k} h^{*}}{E^{*}} \Lambda_{k}+\sum_{k=1}^{N-1} \frac{E^{*}}{h^{*}} \frac{\xi_{k}}{\left(2 \mu_{k}+\lambda_{k}\right)}$,

$\langle d\rangle:=\sum_{k=1}^{N} \frac{2 v_{k}}{\left(1-v_{k}\right)} \frac{\Lambda_{k}}{R_{i}}$.

As an example, consider the homogenization of a periodic laminate made by repeating a group of two layers under imperfect contact. In this case, the "unit cell" of the laminate is $\mathbf{T}_{+} \mathbf{K} \mathbf{T}_{-}$, with $\mathbf{T}_{ \pm}$the transfer matrices of two layers whose elasticity constants are denoted $E_{ \pm}, v_{ \pm}$, and $\hat{\mathbf{K}}$ the matrix characterizes imperfect contact. The thickness ratios $\Lambda_{ \pm}$are assumed to coincide $\Lambda_{+}=\Lambda_{-}=1 / 2$. Thus, 
$\langle a\rangle:=\frac{1}{R_{i}}\left(\frac{\left(1-2 v_{-}\right)}{\left(1-v_{-}\right)}+\frac{\left(1-2 v_{+}\right)}{\left(1-v_{+}\right)}\right)$,

$\langle b\rangle:=\frac{h^{*}}{E^{*} R_{i}^{2}}\left(\frac{E_{-}}{\left(1-v_{-}\right)}+\frac{E_{+}}{\left(1-v_{+}\right)}\right)$,

$\langle c\rangle:=\frac{E^{*}}{h^{*}}\left(\frac{\left(1-2 v_{-}\right)\left(1+v_{-}\right)}{2 E_{-}\left(1-v_{-}\right)}+\frac{\left(1-2 v_{+}\right)\left(1+v_{+}\right)}{2 E_{+}\left(1-v_{+}\right)}+\frac{\xi}{(2 \mu+\lambda)}\right)$,

$\langle d\rangle:=\frac{1}{R_{i}}\left(\frac{v_{-}}{\left(1-v_{-}\right)}+\frac{v_{+}}{\left(1-v_{+}\right)}\right)$.

As a final result, note that comparing the latter relations with the matrix system of a transversely isotropic homogeneous elastic sphere (cf. [22, Eqs. (17)-(19)]) one obtains the equivalent material parameters $E /(1-v), E^{\prime}, v^{\prime}$ of the homogenized sphere consisting of a two-layers laminate with imperfect contact,

$\frac{E}{(1-v)}=\left(\frac{E_{-}}{2\left(1-v_{-}\right)}+\frac{E_{+}}{2\left(1-v_{+}\right)}\right)$

$\frac{1}{E^{\prime}}=\frac{\left(\frac{v_{-}}{\left(1-v_{-}\right)}+\frac{v_{+}}{\left(1-v_{+}\right)}\right)^{2}}{\left(\frac{E_{-}}{\left(1-v_{-}\right)}+\frac{E_{+}}{\left(1-v_{+}\right)}\right)}$

$$
+\frac{\left(1-2 v_{-}\right)\left(1+v_{-}\right)}{2 E_{-}\left(1-v_{-}\right)}+\frac{\left(1-2 v_{+}\right)\left(1+v_{+}\right)}{2 E_{+}\left(1-v_{+}\right)}+\frac{\xi}{(2 \mu+\lambda)},
$$

$\frac{v^{\prime}}{E^{\prime}}=\frac{\left(\frac{v_{-}}{\left(1-v_{-}\right)}+\frac{v_{+}}{\left(1-v_{+}\right)}\right)}{\left(\frac{E_{-}}{\left(1-v_{-}\right)}+\frac{E_{+}}{\left(1-v_{+}\right)}\right)}$.

The state of stresses and displacements of the equivalent transversely isotropic hollow sphere subjected to the boundary conditions (14) and (15) can be obtained by substituting the relations (42)-(44) into Eq. (52) of [22], which are

$$
\begin{aligned}
u_{r}(r) & =\frac{p R_{e}}{\left(\frac{R_{i}}{R_{e}}\right)^{\lambda_{1}-1}-\left(\frac{R_{i}}{R_{e}}\right)^{\lambda_{2}-1}} \frac{h^{*}}{E^{*}}\langle c\rangle\left[\frac{\left(\frac{r}{R_{e}}\right)^{\lambda_{2}}}{R_{i}\langle d\rangle+\lambda_{2}}-\frac{\left(\frac{r}{R_{e}}\right)^{\lambda_{1}}}{R_{i}\langle d\rangle+\lambda_{1}}\right], \\
\sigma_{r r}(r) & =-\frac{p}{\left(\frac{R_{i}}{R_{e}}\right)^{\lambda_{1}-1}-\left(\frac{R_{i}}{R_{e}}\right)^{\lambda_{2}-1}}\left[\left(\frac{r}{R_{e}}\right)^{\lambda_{1}-1}-\left(\frac{r}{R_{e}}\right)^{\lambda_{2}-1}\right], \\
\sigma_{\theta \theta}(r) & =-\frac{p / 2}{\left(\frac{R_{i}}{R_{e}}\right)^{\lambda_{1}-1}-\left(\frac{R_{i}}{R_{e}}\right)^{\lambda_{2}-1}}\left[\left(1+\lambda_{1}\right)\left(\frac{r}{R_{e}}\right)^{\lambda_{1}-1}-\left(1+\lambda_{2}\right)\left(\frac{r}{R_{e}}\right)^{\lambda_{2}-1}\right],
\end{aligned}
$$

with

$$
\begin{aligned}
& \lambda_{1,2}=1 / 2(-1 \pm \sqrt{8 \bar{C}-1}), \\
& \bar{C}=1 / 2\left(R_{i}^{2}\langle b\rangle\langle c\rangle-\left(1-R_{i}\langle d\rangle\right) R_{i}\langle d\rangle\right) .
\end{aligned}
$$

\section{The finite element method}

In this section, a numerical method based on the finite element is proposed to solve problem (6)-(8). Since this technique is quite standard, it is rapidly outlined here.

For the sake of simplicity, we denote $Y_{-}=[0, \gamma)$ and $Y_{+}=(\gamma, 1]$. Then, choosing a test function $v$, which can be discontinuous across the interface $\Gamma^{\varepsilon}$, multiplying the equilibrium Eq. (6) by this test function and integrating among $Y$, one obtains after integration by parts

$\left.-\int_{Y_{-}}\left(C^{i 3 l k}+C^{i 3 m 3} \frac{\partial N_{m}^{l k}}{\partial y} \frac{\partial v}{\partial y}\right) d y+C^{i 3 l k}+C^{i 3 m 3} \frac{\partial N_{m}^{l k}}{\partial y}\right)\left(\gamma^{-}\right) v\left(\gamma^{-}\right)=0$

$$
\left.\left.-\int_{Y_{+}} C^{i 3 l k}+C^{i 3 m 3} \frac{\partial N_{m}^{l k}}{\partial y} \frac{\partial v}{\partial y}\right) d y-C^{i 3 l k}+C^{i 3 m 3} \frac{\partial N_{m}^{l k}}{\partial y}\right)\left(\gamma^{+}\right) v\left(\gamma^{+}\right)=0
$$

Now, adding these two equations, using the continuity of $C^{i 3 l k}+C^{i 3 m 3} \frac{\partial N_{m}^{k k}}{\partial y}$ across the interface, (see Eqs. (7) and (8)), a weak formulation of the problem can be written as follows

$\left.\int_{Y_{ \pm}} C^{i 3 l k}+C^{i 3 m 3} \frac{\partial N_{m}^{l k}}{\partial y} \frac{\partial v}{\partial y}\right) d y+K^{i m}\left[\left[N_{(1) m}^{l k}\right]\right][[v]]=0$

Finally, using standard finite element on each sub domain, and a "flat" finite element on $\Gamma^{\varepsilon}$, that have all its nodes on $\Gamma^{\epsilon}$, the first ones related to $Y_{-}$and the other ones to $Y_{+}$, it is possible to write a rigidity matrix of this problem, that is invertible, with standard error estimation (see [28] or [29] for more details).

Due to finite element discretization, the integrals (see formula (12), for example) for the computation of effective coefficients are substituted by sums over element contributions.

\section{Numerical results}

\subsection{Rectangular shell composite}

Here a flat shell structure is considered in order to validate formula (12). The unit cell is composed of two layers of isotropic materials like aluminum and reinforced carbon fiber. The properties are Young's modulus equal to $150 \mathrm{Gpa}$ and Poisson's ratio 0.3 . The volume fraction of both materials are set to 0.5 . The matrix $\mathbf{K}$ that characterizes the imperfect contact takes the same values as given in Section 3.1. In Table 1 the effective coefficients are obtained using formula (12). The same results are also attained using the formulas for perfect contact homogenized constants (1.19) [4, Chap. 5] and (9.9) [30]. As a particular case, from the model used here of imperfect contact, Eq. (12) gives the same values that formula (1.30) of [4, Chap. 5] with perfect contact (i.e. with very large $K^{11}$ ) for a flat laminated structure.

\subsection{Spherical shell composite}

In contrast to [21] here the imperfect condition is taken as a distribution of linear springs acting at the interface between the two isotropic materials except that now a hollow two layer spherical shell composite is considered with isotropic components.

The inner and outer radius are denoted by $R_{i}=R_{0}-t$ and $R_{e}=R_{0}+t$ respectively, where $t=R_{0} / 10$. The spherical coordinate system $(\theta, \varphi, r)$ is used to describe the geometry of the structure, [12]. The layers of the composite are transversal to the coordinate $r$. The inner surface $r=R_{i}$ of the heterogeneous body is loaded by a constant radial stress, (14), and the external spherical surface $r=R_{e}$ is traction free. The materials used in the composite have the following elastic properties

$\mu_{-}=10 \mu_{+}, \quad \mu=e^{x} \mu_{+}$,

$v_{-}=0.2, \quad v_{+}=0.35, \quad v=0.3$

where $x \in[-3,3]$, the index "-_" denotes the inner layer, the index " +" the outer layer, non-indexed constants are the $\mathbf{K}$ parameters. For this particular case, the matrix $\mathbf{K}$ is diagonal and has components $K^{11}=K^{22}=\mu / \varepsilon$ and $K^{33}=(\lambda+2 \mu) / \varepsilon$.

To obtain the effective elastic properties of the presented spherical shell composite, the two above described approaches, AHM and SAM are used.

As a first step, the local functions $\partial N_{m}^{l k} / \partial y$ are computed via AHM (13) and FEM. The variational formulation (50) and (51) of 
Table 1

Comparison between the effective coefficients obtained via AHM (12) for imperfect contact (I.C.) case and perfect contact (P.C.) condition using the results presented in [4].

\begin{tabular}{|c|c|c|c|c|}
\hline Effective Ceoff. & P.C. & I.C. $K^{11}=1000$ & I.C. $K^{11}=100$ & I.C. $K^{11}=20$ \\
\hline$h^{1111}$ & 157.843037 & 156.2367942 & 146.5355996 & 133.4282785 \\
\hline$h^{1122}$ & 73.46924781 & 71.86300505 & 62.16181048 & 49.05448934 \\
\hline$h^{1133}$ & 71.09793087 & 67.77592879 & 47.71209456 & 20.60377131 \\
\hline$h^{3333}$ & 147.0434479 & 140.1729436 & 98.67728648 & 42.61234521 \\
\hline$h^{1313}$ & 36.48801967 & 35.20351318 & 26.73349628 & 12.91885249 \\
\hline$h^{1212}$ & 42.18689459 & 42.18689459 & 42.18689459 & 42.18689459 \\
\hline
\end{tabular}

the linear system of Eqs. (10) and (11) used to obtain the value of the local function $\partial N_{m}^{l k} / \partial y$ by FEM, reports the exact solution of the system due to the linearity of the system. Thus, a perfect concordance between the local function $\partial N_{m}^{l k} / \partial y$, computed via AHM and FEM, is obtained.

In Table 2, a comparison of the effective coefficients obtained via AHM and FEM using the parameters (52) and (53) and considering $\varepsilon=R_{0} / 100$ is shown. Notice the perfect coincidence between the effective coefficients reported by both methods; this is an expected result since the local functions obtained through AHM and FEM also coincide.

The effective coefficients given in Table 2 are used to obtain the homogenized problem following the methodology described in $[4,14]$. Solving the homogenized problem with the boundary con- ditions (14) and (15), the effective displacement and stress are computed. In order to compare the results obtained by AHM, FEM and the methodology presented in Section 4, the normalized displacement reported in Table 3 is computed using three methods AHM, FEM and SAM for the values of the parameter $x=\{-3,-2,-1,0,1,2,3\}$ at $R_{i}, R_{0}$ and $R_{e}$. Good concordance between the three methods is appreciated.

Considering the effective coefficients of Table 2, the radial displacement of Table 3 and the methodology described in Section 4 , the effective radial $\sigma^{r r}$ and circumferential $\sigma^{\theta \theta}$ stresses are computed using the three methods.

In Table 4, the effective radial stress is computed by AHM, FEM and SAM for different values of the parameter $x$. The good correspondence between the three methods for $r=\left\{R_{i}, R_{e}\right\}$ is due to

Table 2

Values of the effective coefficients $h^{i j k l}$ obtained via AHM and FEM for some values of the parameter $x$.

\begin{tabular}{|c|c|c|c|c|c|c|c|c|}
\hline \multicolumn{9}{|c|}{$h^{i j k l} / \mu_{+}$} \\
\hline \multirow[b]{2}{*}{$x$} & \multicolumn{2}{|l|}{$h^{1111}$} & \multicolumn{2}{|l|}{$h^{1133}$} & \multicolumn{2}{|l|}{$h^{1122}$} & \multicolumn{2}{|l|}{$h^{3333}$} \\
\hline & AHM & FEM & AHM & FEM & $\overline{\mathrm{AHM}}$ & FEM & $\overline{\mathrm{AHM}}$ & FEM \\
\hline-3 & 14.84995 & 14.84995 & 2.05841 & 2.05841 & 3.84995 & 3.84995 & 5.22134 & 5.22134 \\
\hline-2 & 15.03957 & 15.03957 & 2.53939 & 2.53939 & 4.03957 & 4.03957 & 6.44138 & 6.44138 \\
\hline-1 & 15.13372 & 15.13372 & 2.77821 & 2.77821 & 4.13372 & 4.13372 & 7.04716 & 7.04716 \\
\hline 0 & 15.17297 & 15.17297 & 2.87777 & 2.87777 & 4.17297 & 4.17297 & 7.29971 & 7.29971 \\
\hline 1 & 15.18812 & 15.18812 & 2.91622 & 2.91622 & 4.18812 & 4.18812 & 7.39723 & 7.39723 \\
\hline 2 & 15.19380 & 15.19380 & 2.93062 & 2.93062 & 4.19380 & 4.19380 & 7.43377 & 7.43377 \\
\hline 3 & 15.19591 & 15.19591 & 2.93595 & 2.93595 & 4.19591 & 4.19591 & 7.44730 & 7.44730 \\
\hline
\end{tabular}

Table 3

Values of the normalized effective displacement $\mu_{+} u_{r} / p(\cdot)$ obtained via AHM, FEM and SAM for some values of the parameter $x$.

\begin{tabular}{|c|c|c|c|c|c|c|c|c|c|}
\hline \multirow[b]{2}{*}{$x$} & \multicolumn{3}{|l|}{$\mu_{+} u_{r} / p\left(R_{i}\right)$} & \multicolumn{3}{|l|}{$\mu_{+} u_{r} / p\left(R_{0}\right)$} & \multicolumn{3}{|l|}{$\underline{\mu_{+} u_{r} / p\left(R_{e}\right)}$} \\
\hline & AHM & FEM & SAM & AHM & FEM & SAM & AHM & FEM & SAM \\
\hline-3 & -0.15039 & -0.15191 & -0.16851 & -0.12612 & -0.12649 & -0.12857 & -0.11365 & -0.11433 & -0.11224 \\
\hline-2 & -0.14827 & -0.14581 & -0.16224 & -0.12647 & -0.12335 & -0.13398 & -0.11459 & -0.11198 & -0.12014 \\
\hline-1 & -0.14749 & -0.14315 & -0.16218 & -0.12660 & -0.12182 & -0.13813 & -0.11494 & -0.11077 & -0.12510 \\
\hline 0 & -0.14721 & -0.14210 & -0.16310 & -0.12664 & -0.12119 & -0.14054 & -0.11507 & -0.11026 & -0.12775 \\
\hline 1 & -0.14710 & -0.14170 & -0.16367 & -0.12666 & -0.12094 & -0.14164 & -0.11511 & -0.11007 & -0.12893 \\
\hline 2 & -0.14706 & -0.14155 & -0.16393 & -0.12667 & -0.12085 & -0.14208 & -0.11513 & -0.10999 & -0.12939 \\
\hline 3 & -0.14705 & -0.14150 & -0.16403 & -0.12667 & -0.12082 & -0.14225 & -0.11514 & -0.10997 & -0.12957 \\
\hline
\end{tabular}

Table 4

Values of the normalized effective radial stress $\sigma_{r r} / p(\cdot)$ obtained via AHM, FEM and SAM for some values of the parameter $x$.

\begin{tabular}{|c|c|c|c|c|c|c|c|c|c|}
\hline \multirow[b]{2}{*}{$x$} & \multicolumn{3}{|c|}{$\sigma_{r r} / p\left(R_{i}\right)$} & \multicolumn{3}{|l|}{$\sigma_{r r} / p\left(R_{0}\right)$} & \multicolumn{3}{|c|}{$\sigma_{r r} / p\left(R_{e}\right)$} \\
\hline & AHM & FEM & SAM & AHM & FEM & SAM & AHM & FEM & SAM \\
\hline-3 & 1 & 1 & 1 & 0.39194 & 0.40777 & 0.37774 & 0 & 0 & 0 \\
\hline-2 & 1 & 1 & 1 & 0.39442 & 0.41001 & 0.38967 & 0 & 0 & 0 \\
\hline-1 & 1 & 1 & 1 & 0.39535 & 0.41086 & 0.39422 & 0 & 0 & 0 \\
\hline 0 & 1 & 1 & 1 & 0.39569 & 0.41117 & 0.39591 & 0 & 0 & 0 \\
\hline 1 & 1 & 1 & 1 & 0.39581 & 0.41129 & 0.39654 & 0 & 0 & 0 \\
\hline 2 & 1 & 1 & 1 & 0.39586 & 0.41133 & 0.39677 & 0 & 0 & 0 \\
\hline 3 & 1 & 1 & 1 & 0.39588 & 0.41135 & 0.39685 & 0 & 0 & 0 \\
\hline
\end{tabular}


Table 5

Values of the normalized effective radial stress $\sigma_{\theta \theta} / p(\cdot)$ obtained via AHM, FEM and SAM for some values of the parameter $x$.

\begin{tabular}{|c|c|c|c|c|c|c|c|c|c|}
\hline \multirow[b]{2}{*}{$x$} & \multicolumn{3}{|l|}{$\sigma_{\theta \theta} / p\left(R_{i}\right)$} & \multicolumn{3}{|l|}{$\sigma_{\theta \theta} / p\left(R_{0}\right)$} & \multicolumn{3}{|l|}{$\sigma_{\theta \theta} / p\left(R_{e}\right)$} \\
\hline & AHM & FEM & SAM & AHM & FEM & SAM & AHM & FEM & SAM \\
\hline-3 & 2.45936 & 2.48818 & 2.69206 & 1.99925 & 1.99922 & 1.97031 & 1.76450 & 1.77485 & 1.68188 \\
\hline-2 & 2.41921 & 2.37235 & 2.49624 & 2.00421 & 1.94473 & 1.99469 & 1.77900 & 1.73844 & 1.75124 \\
\hline-1 & 2.40438 & 2.32194 & 2.42263 & 2.00604 & 1.91827 & 2.00379 & 1.78437 & 1.71966 & 1.77776 \\
\hline 0 & 2.39891 & 2.30199 & 2.39533 & 2.00672 & 1.90737 & 2.00716 & 1.78636 & 1.71178 & 1.78766 \\
\hline 1 & 2.39690 & 2.29444 & 2.38526 & 2.00696 & 1.90319 & 2.00840 & 1.78709 & 1.70873 & 1.79132 \\
\hline 2 & 2.39616 & 2.29163 & 2.38155 & 2.00706 & 1.90163 & 2.00886 & 1.78736 & 1.70759 & 1.79267 \\
\hline 3 & 2.39589 & 2.29059 & 2.38019 & 2.00709 & 1.90105 & 2.00902 & 1.78746 & 1.70716 & 1.79316 \\
\hline
\end{tabular}

the boundary conditions (14) and (15). In Table 5, the effective circumferential stress is reported for the spherical structure mentioned above. The results have similar behavior for the three methods and the same values of the parameter $x$.

It can be seem that the results presented in Tables 3-5 for the linear spring model, coincide with the values obtained with model where the imperfect contact condition is idealized by a thin interphase between the layers of the composite, i.e. a three phase composite as it is given in [21].

\section{Conclusions}

In this paper three different approaches areused to study the elastic properties of a spherical shell composite. The two-scale Asymptotic Homogenization Method is used to obtain the general expression of the local problems and the effective coefficients of elastic composites with imperfect contact at the interface. The expression of such effective coefficients is given in (12). The results are compared for different cases of imperfections and the limit case reported in [14] for perfect contact is derived, considering a particular composite. The methodology for spherical shell composites with imperfect contact at the interface is implemented. The local problems for this structure are solved analytically and via FEM. The solution of local functions are used to compute the effective coefficients, and a good coincidence between AHM and FEM is appreciated. Moreover, a third method considering the spherical assemblage model (SAM) is proposed and the general expression for the elastic properties of a spherical structure with imperfect contact at the interface is derived. The general expression, via SAM, of the displacement, radial and circumferential stresses for the spherical structure are given in the Eqs. (45)-(47). Comparison of the effective displacement, radial and circumferential stresses obtained via AHM, FEM and SAM show good results.

\section{Acknowledgements}

The authors gratefully acknowledge to the project SHICHAN, supported by FSP (Cooperation Scientifique Franco-Cubaine) PROJET No $29935 \mathrm{XH}$ and to the project Composite Materials from University of Havana. SHIHMAS Interfaces rigides et souples dans les matriaux et structures htrognes (Soft and Hard Interfaces for Heterogeneous Materials and Structures), 2016 sponsored by French Embassy in La Habana is also grateful. Thanks to Departamento de Matemáticas y Mecánica, IIMAS-UNAM, for its support and Ramiro Chávez Tovar and Ana Pérez Arteaga for computational assistance.

\section{References}

[1] Guinovart-Díaz R, Rodríguez-Ramos R, López-Realpozo JC, Bravo-Castillero J Otero JA, Sabina FJ, Lebon F, Dumont S. Analysis of fibrous elastic composites with nonuniform imperfect adhesion. Acta Mechanica 2016. http://dx.doi.org/ 10.1007/s00707-015-1428-X.
[2] Rodríguez-Ramos R, Guinovart-Díaz R, López-Realpozo JC, Bravo-Castillero J, Otero JA, Sabina FJ. Influence of imperfect elastic contact condition on the antiplane effective properties of piezoelectric fibrous composites. Arch Appl Mech 2010;80:377-88. http://dx.doi.org/10.1007/s00419-009-0320-3.

[3] Rizzoni R, Lebon F. Asymptotic analysis of an adhesive joint with mismatch strain. Eur J Mech - A/Solids 2012;36:1-8. http://dx.doi.org/10.1016/i. euromechsol.2012.02.005. URL http://www.sciencedirect.com/science/article/ pii/S0997753812000290.

[4] Pobedrya B. Mechanics of composite materials. Moscow, Russia: Moscow State University Press: 1984.

[5] Bakhvalov N, Panasenko G. Homogenisation: Averaging Processes in Periodic Media, Mathematics and its Application (Soviet Series); 1989.

[6] Guinovart-Díaz R, Rodríguez-Ramos R, Bravo-Castillero J, Sabina FJ, CamachoMontes H. Electro-mechanical moduli of three-phase fiber composites. J Mater Lett 2008;262:2385-7. http://dx.doi.org/10.1016/i.matlet.2007.12.007.

[7] López-Realpozo JC, Rodríguez-Ramos R, Guinovart-Díaz R, Bravo-Castillero J, Sabina FJ. Transport properties in fibrous elastic rhombic composite with imperfect contact condition. Int J Mech Sci 2011;53:98-107. http://dx.doi.org/ 10.1016/i.ijmecsci.2010.11.006.

[8] Otero JA, Rodríguez-Ramos R, Bravo-Castillero J, Guinovart-Díaz R, Sabina FJ, Monsivais G. Semi-analytical method for computing effective properties in elastic composite under imperfect contact. Int J Solids Struct 2013;50:609-22. http://dx.doi.org/10.1016/j.ijsolstr.2012.11.001.

[9] Guinovart-Díaz R, Rodríguez-Ramos R, Bravo-Castillero J, López-Realpozo J, Sabina F, Sevostianov I. Effective elastic properties of a periodic fiber reinforced composite with parallelogram-like arrangement of fibers and imperfect contact between matrix and fibers. Int J Solids Struct 2013;50(13):2022-32. http://dx.doi.org/10.1016/i.ijsolstr.2013.02.019. URL http:// www.sciencedirect.com/science/article/pii/S0020768313000905.

[10] Lebon F, Rizzoni R. Asymptotic analysis of an adhesive joint: a focus on cylindrical coordinates. Machine Dyn Res 2011;35:97-107.

[11] Tsalis D, Chatzigeorgiou G, Charalambakis N. Homogenization of structures with generalized periodicity. Compos: Part B 2012;43:2495-512.

[12] Rizzoni R, Lebon F. Imperfect interfaces as asymptotic models of thin curved elastic adhesive interphases. Mech Res Commun 2013;51:39-50. http://dx.doi. org/10.1016/i.mechrescom.2013.04.008.

[13] Strek T, Jopek H, Maruszewski B, Nienartowicz M. Computational analysis of sandwich-structured composites with an auxetic phase. Phys Status Solidi B 2014;251:354-66.

[14] Guinovart-Sanjuán D, Rodríguez-Ramos R, Guinovart-Díaz R, Bravo-Castillero J, Sabina FJ, Merodio J, Lebon F, Dumont S, Conci A. Effective properties of regular elastic laminated shell composite. Compos Part B 2016;87:12-20. http://dx.doi.org/10.1016/i.compositesb.2015.09.051.

[15] Hashin Z. Thin interphaseimperfect interface in conduction. J Appl Phys 2001;89:2261-7. http://dx.doi.org/10.1063/1.1337936.

[16] Hashin Z. Thin interphase/imperfect interface in elasticity with application to coated fiber composites. J Mech Phys Solids 2002;50:2509-37.

[17] López-Realpozo JC, Rodríguez-Ramos R, Guinovart-Díaz R, Bravo-Castillero J, Otero JA, Sabina FJ, Lebon F, Dumont S, Sevostianov I. Effective elastic shear stiffness of a periodic fibrous composite with non-uniform imperfect contact between the matrix and the fibers. Int J Solids Struct 2014;51:1253-62. http:// dx.doi.org/10.1016/i.ijsolstr.2013.12.015.

[18] Chen YC, Rajagopal KR, Wheeler L. Homogenization and global responses of inhomogeneous spherical nonlinear elastic shells. J Elasticity 2006;82:193-214.

[19] Gager J, Pettermann H. Fem modeling of multilayered textile composites based on shell elements. Compos Part B 2015;77:46-51.

[20] Dumont S, Lebon F, Ould-Khaoua A. A numerical tool for periodic heterogeous media, application to interface in al/sic composite. J Appl Mech 2000;67 (1):214-7.

[21] Guinovart-Sanjuán D, Rizzoni R, Rodríguez-Ramos R, Guinovart-Díaz R, BravoCastillero J, Alfonso-Rodriguez R, Lebon F, Dumont S, Sabina FJ. Assessment of models and methods for pressurized spherical composites. Math Mech Solids 2016;87:1-12. http://dx.doi.org/10.1177/1081286516673233.

[22] Bufler H. The arbitrarily and the periodically laminated elastic hollow sphere: exact solutions and homogenization. Arch Appl Mech 1998;68:579-88.

[23] Pestel E, Leckie F. Matrix methods in elastomechanics. New York, San Francisco, Toronto, London: McGraw-Hill; 1963. 
[24] Benveniste Y, Miloh T. Imperfect soft and stiff interfaces in two-dimensional elasticity. Mech Mater 2011;33:309-23.

[25] Rizzoni R, Dumont S, Lebon F, Sacco E. Higher order model for soft and hard elastic interfaces. Int J Solids Struct 2014;51:4137-48.

[26] Klarbring A. Derivation of the adhesively bonded joints by the asymptotic expansion method. Int J Eng Sci 1991;29:493-512.

[27] Geymonat G, Krasucki F, Lenci S. Mathematical analysis of a bonded joint with a soft thin adhesive. Math Mech Solids 1999;4:201-25.
[28] Nairn J. Numerical implementation of imperfect interfaces. Comp Mat Sci 2007; 40:525-36.

[29] Dumont S, Lebon F, Rizzoni R. An asymptotic approach to the adhesion of thin stiff films. Mech Res Commun 2014;58:24-35.

[30] Milton G. The theory of composites, cambridge monographs on applied and computational mathematics. Cmabridge University Press; 2004. 